\title{
BMJ
}

\section{Effectiveness of quinine versus artemether-lumefantrine for treating uncomplicated falciparum malaria in Ugandan children: randomised trial}

\author{
Jane Achan, lecturer, ${ }^{1}$ James K Tibenderana, epidemiologist, ${ }^{2}$ Daniel Kyabayinze, epidemiologist, ${ }^{3}$ Fred \\ Wabwire Mangen, professor, ${ }^{4}$ Moses R Kamya, professor, ${ }^{4}$ Grant Dorsey, professor, ${ }^{5}$ Umberto D’Alessandro, \\ professor, ${ }^{6}$ Philip J Rosenthal, professor, ${ }^{5}$ Ambrose 0 Talisuna, epidemiologist ${ }^{4,6}$
}

\section{${ }^{1}$ Makerere University School of Health Sciences, PO Box 7475 , Kampala, Uganda \\ ${ }^{2}$ Malaria Consortium, Uganda, London School of Hygiene and Tropical Medicine \\ ${ }^{3}$ COMDIS Research Programme Consortium, Malaria Consortium, Uganda \\ ${ }^{4}$ Makerere University School of Health Sciences, Uganda Malaria Surveillance Project \\ ${ }^{5}$ Department of Medicine University of California San Francisco \\ ${ }^{6}$ Institute of Tropical Medicine, Antwerp, Belgium \\ Correspondence to: J Achan \\ achanj@yahoo.co.uk}

Cite this as: $B M J$ 2009;339:b2763 doi:10.1136/bmi.b2763

\section{ABSTRACT}

Objective To compare the effectiveness of oral quinine with that of artemether-lumefantrine in treating uncomplicated malaria in children.

Design Randomised, open label effectiveness study. Setting Outpatient clinic of Uganda's national referral hospital in Kampala.

Participants 175 children aged 6 to 59 months with uncomplicated malaria.

Interventions Participants were randomised to receive oral quinine or artemether-lumefantrine administered by care givers at home.

Main outcome measures Primary outcomes were parasitological cure rates after 28 days of follow-up unadjusted and adjusted by genotyping to distinguish recrudescence from new infections. Secondary outcomes were adherence to study drug, presence of gametocytes, recovery of haemoglobin concentration from baseline at day 28 , and safety profiles.

Results Using survival analysis the cure rate unadjusted by genotyping was $96 \%$ for the artemether-lumefantrine group compared with $64 \%$ for the quinine group (hazard ratio $10.7,95 \%$ confidence interval 3.3 to $35.5, P=0.001$ ). In the quinine group $69 \%(18 / 26)$ of parasitological failures were due to recrudescence compared with none in the artemether-lumefantrine group. The mean adherence to artemether-lumefantrine was $94.5 \%$ compared with $85.4 \%$ to quinine $(P=0.0008)$. Having adherence levels of $80 \%$ or more was associated with a decreased risk of treatment failure $(0.44,0.19$ to $1.02, \mathrm{P}=0.06)$. Adverse events did not differ between the two groups.

Conclusions The effectiveness of a seven day course of quinine for the treatment of uncomplicated malaria in Ugandan children was significantly lower than that of artemether-lumefantrine. These findings question the advisability of the recommendation for quinine therapy for uncomplicated malaria in Africa.

Trial registration ClinicalTrials.gov NCT00540202.

\section{INTRODUCTION}

Most episodes of falciparum malaria are uncomplicated and treated with short courses of oral antimalarials. Chloroquine was the mainstay of therapy for uncomplicated falciparum malaria until the late $1990 \mathrm{~s}$. As a result of the increasing resistance to chloroquine, sulfadoxine-pyrimethamine or amodiaquine was adopted as first line therapy in several countries. However, increasing resistance to both of these drugs has led to recommendations for combination therapy, preferably those based on artemisinin. ${ }^{1}$ Consequently artemether-lumefantrine or artesunate-amodiaquine have been adopted as first line regimens for uncomplicated falciparum malaria in nearly all countries in Africa.

Quinine was the first established antimalarial, and it has been used to treat malaria for centuries. ${ }^{2}$ Intravenous quinine is the standard therapy for severe falciparum malaria in all African countries. Many malaria endemic countries that recently adopted artemisinin based combination therapies as first line therapy recommend quinine as the second line regimen for uncomplicated malaria despite guidelines from the World Health Organization that antimalarial medicines should be used in combination. ${ }^{3}$ Probably such a choice was influenced by the few alternatives to quinine at the time of the policy change to artemisinin based combination therapy. Of the 41 African countries that have adopted artemisinin based combination therapies as the first line treatment for uncomplicated malaria, 29 currently recommend quinine as second line therapy. ${ }^{4}$ In addition, as a result of the decreased efficacy of older agents and limited availability of artemisinin based combination therapies, quinine is increasingly used as a first line drug in the treatment of uncomplicated malaria in Africa. A survey in Uganda in 2007 reported that in rural health facilities quinine was prescribed for only $4 \%$ of patients with uncomplicated malaria ${ }^{5}$ whereas at the outpatient facility of Mulago Hospital, Kampala, this proportion was as high as $26 \%$ (unpublished data). Thus, although it is not listed as a first line drug for uncomplicated malaria in any country, quinine is still widely used for this purpose. 
In Uganda the current and previous national malaria treatment policy recommends quinine, given in its tablet formulation, as the second line treatment for uncomplicated malaria. This recommendation is based on the longstanding assumption that the medicine is efficacious, given the lack of historical and contemporary evidence to the contrary. Although there are anecdotal reports that its effectiveness is compromised by poor adherence to treatment, no reliable evidence shows this. Indeed it was for these reasons that we carried out this study.

Despite increasing use of quinine, few studies of its efficacy in the management of uncomplicated malaria have been published recently. In particular, few studies have used modern methods to determine outcomes (at least 28 days of follow-up and molecular genotyping to distinguish recrudescence from new infections after treatment). Treatment of uncomplicated malaria with a shorter course $(<7$ days) of quinine was generally inferior to a treatment course of seven days. ${ }^{6}$ Considering seven day courses, available recent studies have shown failure rates with quinine for uncomplicated malaria of over $10 \%$ at 28 days in $\operatorname{Sudan}^{7}$ and Thailand. ${ }^{8}$ Quinine efficacy may be particularly poor in pregnant women; both genotype adjusted efficacy after supervised seven day therapy in Thailand ${ }^{9}$ and unadjusted effectiveness after seven day therapy in Gabon ${ }^{10}$ were only about $60 \%$. However, other studies have shown excellent efficacy for quinine, including over $95 \%$ success after 28 days for the treatment of falciparum malaria in Equatorial Guinea, ${ }^{11}$ Venezuela, ${ }^{12}$ and returned travellers in France. ${ }^{13}$

As study designs have varied, it is difficult to ascertain if limitations in quinine effectiveness in some studies were due principally to true drug resistance, varied pharmacokinetics, poor compliance with a seven day regimen, or a combination of these factors. However, available evidence suggests that African strains of Plasmodium falciparum generally remain sensitive to quinine ${ }^{14-19}$ and that variability in quinine pharmacokinetics does not explain varied treatment responses. ${ }^{20}$ Thus poor compliance with a seven day quinine regimen might contribute importantly to limitations in the drug's efficacy. Poor compliance with quinine, compared with shorter regimens, has been documented. ${ }^{21}$ Inadequate compliance is probably due both to the need for frequent dosing over one week and to known poor tolerance of quinine, which often causes the syndrome of cinchonism, including nausea, headache, tinnitus, and blurred vision ${ }^{22}$ at treatment doses. Considering the uncertain effectiveness of quinine and its increasing role in the treatment of uncomplicated malaria in Africa, we compared its effectiveness with that of artemether-lumefantrine, the new first line therapy for uncomplicated malaria in Uganda.

\section{METHODS}

This was an open label randomised effectiveness study to compare parasitological and clinical cure rates and adherence between quinine and artemether-lumefantrine in children with uncomplicated malaria.
Participants were recruited from the main outpatient clinic at Uganda's national referral hospital, Mulago, in Kampala.

Children aged 6 to 59 months were included if they had an axillary temperature of $37.5^{\circ} \mathrm{C}$ or more or a history of fever in the past 24 hours, microscopically confirmed $P$ falciparum mono-infection with any density of asexual parasites, ability to tolerate oral therapy, and no history of antimalarial drug intake in the preceding two weeks. Children were not recruited if they met at least one of several exclusion criteria: a history of allergy to quinine or artemether-lumefantrine, evidence of severe malaria or other concomitant febrile illness, or residence more than $20 \mathrm{~km}$ from the health clinic. Severe malaria was defined according to WHO criteria. $^{23}$

\section{Enrolment procedures}

Participants with suspected malaria during an outpatient visit (day 0) were assessed for eligibility and referred to the laboratory to obtain thick (parasite density) and thin (parasite species) blood smears. Participants fulfilling all selection criteria had a standard baseline history and physical examination done by a study doctor. Study data were recorded on case record forms. Participants were identified by their initials and study identification numbers. Haemoglobin was measured from finger prick blood samples using a portable spectrophotometer (HemoCue; Angelhom, Sweden). Blood samples were also stored on Whatmann filter paper for subsequent molecular studies.

\section{Randomisation and treatment}

Participants were randomly assigned to receive oral quinine or artemether-lumefantrine. Block randomisation was used with blocks of 20. Computer generated randomisation codes were prepared by an independent individual and enclosed in sequentially numbered opaque sealed envelopes, each of which contained the treatment allocation. The envelopes were assigned in sequential order to participants after inclusion. Participants in the quinine arm received a seven day course of quinine sulphate as $10 \mathrm{mg} / \mathrm{kg}$ body weight per dose three times daily. Quinine sulphate was provided as $300 \mathrm{mg}$ tablets (Rene Pharmaceutical, Kampala, Uganda); the quality of the drug was certified by the Uganda National Drug Authority. The tablets were divided when necessary to adjust the dosage for body weight. Care givers were instructed by the study nurse that the tablets should be taken with clean water every eight hours. Participants in the artemether-lumefantrine arm received $\mathrm{WHO}$ recommended weight specific artemether-lumefantrine blister packs (Coartem; Novartis Pharma, Basel, Switzerland): one tablet per dose for body weight $10.0-14.9 \mathrm{~kg}$, two tablets for 15.0-24.9 kg, three tablets for 25.0-34.9 kg, and four tables for $\geq 35 \mathrm{~kg}$. Care givers were instructed by the study nurse to administer a dose of artemether-lumefantrine eight hours after the first dose and then each morning and evening for the following two days, with all doses given 30 minutes to one hour after food, 


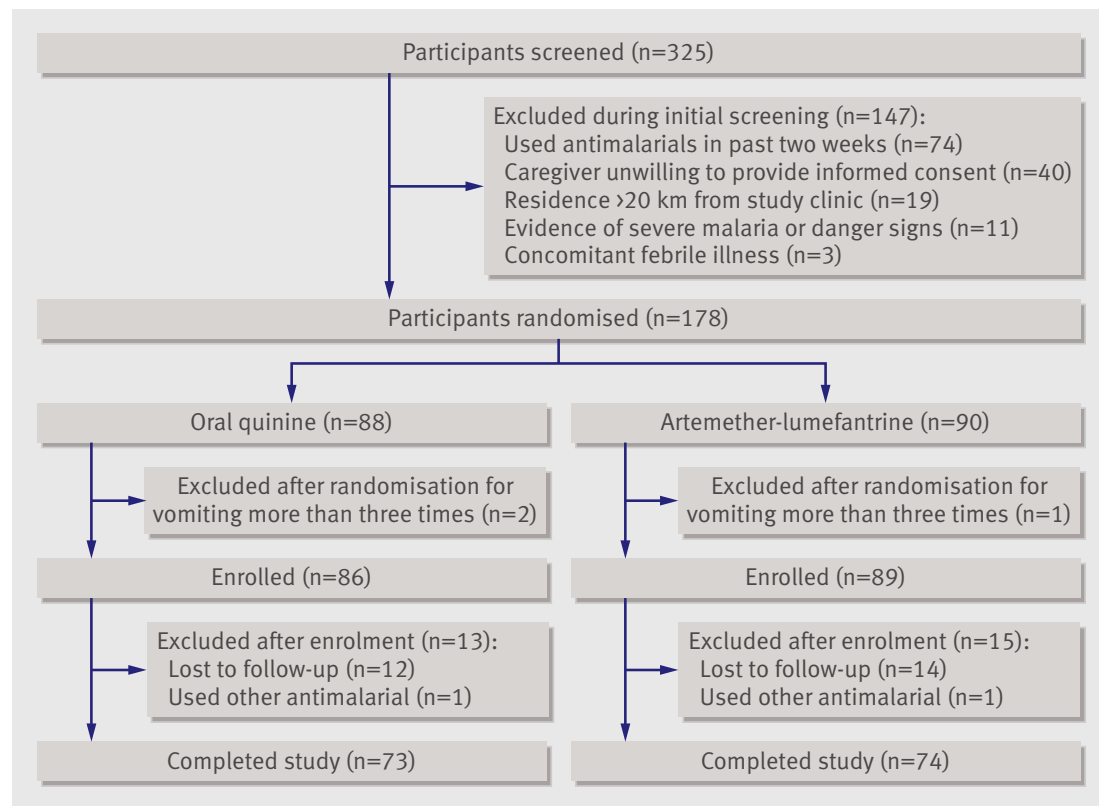

Fig 1| Trial profile up visits scheduled for days 7, 14, and 28 for both groups. Participants were also seen at the clinic on any unscheduled day if they were unwell. On each of these clinic visits a standardised history and physical examination were done and blood smears obtained by finger prick to determine the density of asexual parasites, presence of gametocytes, and haemoglobin concentration. Blood was also collected on filter paper for subsequent molecular studies. In the event of treatment failure, thin smears were examined for species of malaria parasite. Participants who failed treatment were given dihydroartemisinin plus piperaquine phosphate (Duo-cotexcin; Beijing Holley-Cotec, Beijing, China) once daily for three days. Participants who developed severe malaria were referred for treatment with intravenous quinine. Those who for any reason could not continue with the prescribed drugs, developed a serious concomitant illness that interfered with outcome classification, could not be located within 48 hours of a scheduled visit, or had consent withdrawn were included in the analysis and censored on their last day of follow-up.

Adverse events, defined as any unfavourable or undesirable medical occurrence, were assessed at each visit and recorded on case record forms. Association of adverse events with the study drugs was classified as definite, probable, possible, unlikely, or none; according to WHO and the guidelines of the International Conference on Harmonisation. ${ }^{24}$ Adverse events were graded as serious if they were fatal or life threatening, resulted in prolonged hospital stay, caused persistent disability or incapacity, or required medical or surgical interventions to prevent serious outcomes.

\section{Definition of adherence}

Adherence to treatment was assessed by care givers' report, and pill counts; overall adherence was the percentage of prescribed pills taken. For the artemetherlumefantrine arm additional components to assess adherence included an empty blister pack, a pill count on the basis of tablets remaining in the blister pack, care givers' reported dosing schedule, and care givers' report on whether tablets were given after food. On the basis of these factors, adherence to artemetherlumefantrine was further qualified as optimal adherence (all four components present), good adherence (any three components present), or non-adherence (two or fewer components present).

\section{Laboratory procedures}

The density of asexual malaria parasites was determined from thick blood smears stained with $2 \%$ Giemsa for 30 minutes and a count of the number of parasites per 200 white blood cells. Results were expressed as number of parasites per microlitre, assuming a total white cell count of 8000 per microlitre. When no asexual forms were found on examination of 100 high power fields, a negative result was recorded. All slides were read by a second microscopist, and a 
Table 1|Baseline characteristics of children with uncomplicated malaria randomised to receive quinine or artemether-lumefantrine. Values are means (standard deviations) unless stated otherwise

\begin{tabular}{lcc} 
Characteristic & $\begin{array}{c}\text { Quinine group } \\
(\mathrm{n}=86)\end{array}$ & $\begin{array}{c}\text { Artemether-lumefantrine group } \\
(\mathrm{n}=89)\end{array}$ \\
Age (years) & $2.0(1.5)$ & $2.1(1.4)$ \\
\hline No $(\%)$ girls & $45(52)$ & $49(55)$ \\
\hline Weight $(\mathrm{kg})$ & $11.3(3.3)$ & $11.3(3.0)$ \\
\hline Axillary temperature $\left({ }^{\circ} \mathrm{C}\right)$ & $37.8(1.4)$ & $37.7(1.3)$ \\
\hline Haemoglobin concentration $(\mathrm{g} / \mathrm{l})$ & $97(20)$ & $96(21)$ \\
\hline No $(\%)$ with gametocytes present & $7(8)$ & $10(11)$ \\
\hline Parasite density (geometric mean $/ \mu \mathrm{l})$ & 14107 & 16124 \\
\hline No $(\%)$ with parasitaemia $<5000 / \mu \mathrm{l}$ & $24(28)$ & $22(25)$ \\
\hline
\end{tabular}

third reviewer settled any discrepancies. Microscopists were blinded to treatment assignments.

For participants with recurrent parasitaemia after day 3, we extracted DNA using chelex from filter paper blood samples collected at enrolment and on the day of failure. ${ }^{25}$ To distinguish between recrudescence and new infection, samples from enrolment and the day of treatment failure were compared in a stepwise manner on the basis of polymorphisms in merozoite surface protein-1, merozoite surface protein-2, and four microsatellite markers (TA40, TA60, TA81, and PfPK2). ${ }^{26}$

\section{Outcome classification}

Treatment outcomes were assessed according to WHO guidelines as adequate clinical and parasitological response, early treatment failure, late clinical failure, or late parasitological failure. ${ }^{27}$ The primary effectiveness end point was the polymerase chain reaction adjusted clinical and parasitological cure rate at day 28; secondary effectiveness outcomes were polymerase chain reaction unadjusted cure rate at day 28 , adherence to treatment regimens, presence of gametocytes, recovery of haemoglobin concentration from baseline at day 28 , and incidence of adverse events.

\section{Sample size estimation}

This study was designed to test the hypothesis that the effectiveness of quinine and artemether-lumefantrine would not differ in the treatment of uncomplicated malaria. We calculated that 151 patients would be needed in each treatment arm using a $5 \%$ level of significance and $90 \%$ power, and assuming a difference in adherence between the two groups of $19 \% \%^{2128}$ and a $10 \%$ loss to follow-up.

\section{Statistical analysis}

Data were double entered into EPI-info software version 6.04 and analysed using Stata version 10.0. Effectiveness analysis was done using a modified intention to treat with survival analysis. Categorical data were compared by $\chi^{2}$ test or Fisher's exact test. Differences between group means were analysed using Student's $t$ test, applying log transformation when appropriate. Risks of treatment failure were estimated using
Kaplan-Meier survival analysis. Two tailed $\mathrm{P}$ values were used and all analyses were done at a 5\% significance level.

\section{RESULTS}

Between September 2007 and April 2008 a total of 325 children were screened for eligibility; of these, 147 $(46 \%)$ were excluded, most commonly for use of antimalarials in the two weeks before enrolment or unwillingness of the primary care givers to provide informed consent (fig 1). A planned interim analysis was undertaken by the data and safety monitoring board after 178 participants had been recruited. The oral quinine group met the predefined stopping rule of a significant difference in cure rate using the O'Brien Fleming method, with $\mathrm{P}<0.001$, and the study was halted.

Eighty nine participants were enrolled to receive artemether-lumefantrine and 86 to receive quinine. During follow-up, 28 of the 175 participants $(16 \%)$ were excluded, 13 in the quinine arm (12 lost to follow-up and one took other antimalarial) and 15 in the artemether-lumefantrine arm (14 lost to follow-up and one took other antimalarial). These participants were included in the analysis and censored on their last day of follow-up. Thus primary outcomes (unadjusted and adjusted by genotyping) were available for $85 \%$ (73/ 86) of participants in the quinine arm and $83 \%$ (74/ 89) in the artemether-lumefantrine arm. Baseline characteristics were comparable between the groups (table 1).

\section{Primary treatment outcomes}

Cure rates were significantly higher in the artemetherlumefantrine group than in the quinine group. Using survival analysis, cure rates unadjusted by genotyping were $96 \%$ for the artemether-lumefantrine group and $64 \%$ for the quinine group $(\mathrm{P}<0.001$; table 2$)$. Early treatment failure was uncommon; only two cases were seen, both in the quinine arm (one child had more than two convulsions in 24 hours and the other had profuse vomiting and prostration). They were both admitted to hospital and treated with intravenous quinine. Participants were 10 times more likely to fail treatment with oral quinine than with artemetherlumefantrine (hazard ratio 10.7, 95\% confidence interval 3.3 to $35.5, \mathrm{P}=0.001$ ). The risk of treatment failure unadjusted by genotyping was significantly higher in the quinine group than in the artemether-lumefantrine group (35.3\%, 95\% confidence interval $25.6 \%$ to $47.4 \%$ $v 4.1 \%, 1.3 \%$ to $12.0 \%)$ : risk difference $31.3 \%(19.4 \%$ to $31.1 \%, \mathrm{P}<0.001$; table 3 , fig 2$)$. Genotyping showed that $62 \%(16 / 26)$ of treatment failures in the quinine group were due to recrudescence and that all treatment failures in the artemether-lumefantrine group were due to new infections. When only treatment failures caused by recrudescent parasites were considered, the risk of failure with quinine was $23.1 \%$ (95\% confidence interval $14.9 \%$ to $35.0 \%$ ) and with artemether-lumefantrine was 0 (risk difference $23.1 \%, 13.2 \%$ to $33.1 \%, \mathrm{P}<0.001$; table 3). The predictors of treatment failure using Cox regression analysis included treatment with oral 
Table 2 Treatment outcomes of children aged 6 to 59 months with uncomplicated malaria after 28 days of follow-up. Values are percentages (numbers)

\begin{tabular}{lcc} 
Treatment outcomes & Quinine group (n=86) & Artemether-lumefantrine group (n=89) \\
Cure rate* & $64(47)$ & $97(71)$ \\
\hline Early treatment failure† & $3(2)$ & 0 \\
\hline Late clinical failuret: & & 0 \\
\hline Due to recrudescence & $12(10)$ & $2(2)$ \\
\hline Due to new infection & $2(5)$ & 0 \\
\hline Genotyping unsuccessful & 0 & $3(2)$ \\
\hline Total & $21(15)$ & 0 \\
\hline Late parasitological failure†: & & $1(1)$ \\
\hline Due to recrudescence & $2(6)$ & 0 \\
\hline Due to new infection & $2(2)$ & $1(1)$ \\
\hline Genotyping unsuccessful & $1(1)$ & $16(14)$ \\
\hline Total & $7(9)$ & $1(1)$ \\
\hline No treatment outcome: & & $17(15)$ \\
\hline Lost to follow-up & $14(12)$ & \\
\hline Use of other antimalarials & $1(1)$ &
\end{tabular}

*Adequate clinical and parasitological response.

†Concerns 73 children in quinine arm and 74 in artemether-lumefantrine arm in whom treatment outcome was assessed. quinine (hazard ratio $11.06,95 \%$ confidence interval 3.34 to $36.57, \mathrm{P}<0.001)$ and a temperature greater than $37.5^{\circ} \mathrm{C}$ on day $0(2.57,1.17$ to $5.66, \mathrm{P}=0.02)$.

\section{Secondary outcomes}

Certain early responses to treatment, including clearance of fever and parasites, could not be assessed in this study because participants were only seen at the study clinic on days 0 and 7. Haemoglobin concentrations improved equally in both groups during 28 days of follow-up. Gametocytaemia was more common in the quinine group at day 7 compared with the artemether-lumefantrine group (14\% (10/73) v 1\% (1/74); $\mathrm{P}=0.001)$. By day 28 the groups did not differ (table 4 ). Total person time with gametocytes was 20 weeks for quinine compared with five weeks for artemetherlumefantrine $(\mathrm{P}<0.01)$.

Reported adverse events did not differ between the groups. Common side effects of quinine such as nausea, headache, tinnitus, and blurred vision were not noted. Severe adverse events occurred in only three participants, two in the quinine group (one participant had repeated seizures and the other had recurrent vomiting and prostration). They were both classified as early treatment failures. The third participant, in the artemether-lumefantrine group, had repeated vomiting on day 7 and a negative malaria smear result at that time. This participant had a urinary tract infection and improved with antibiotic therapy.

\section{Adherence}

Adherence was assessed in 91\% (160/175) of participants; 75 in the quinine group and 85 in the artemether-lumefantrine group. This was done successfully at the first home visit in $86 \%(n=138)$ of the participants and on a second home visit in $12 \%$ $(\mathrm{n}=19)$. Respondents were predominantly mothers
$(94 \%, \mathrm{n}=150)$ who were unemployed and stayed at home $(60 \%, \mathrm{n}=96) ; 56 \%(\mathrm{n}=89)$ were taking care of at least two children and $35 \%(n=56)$ were taking care of three or four children. Ninety per cent $(n=144)$ of these care givers had at least a primary school education.

Mean adherence in the artemether-lumefantrine group was 95\% (range 33-100\%) and in the quinine group was $85 \%$ (range $14-100 \% ; \mathrm{P}=0.0008$ ). Overall, non-adherence to treatment was higher in the quinine group than in the artemether-lumefantrine group $(55 \%$ $(41 / 75) v 17 \%(14 / 85) ; \mathrm{P}=0.001)$. On the third day of treatment the proportion of patients not adhering to treatment was similar between the groups; $12 \%$ in the artemether-lumefantrine arm and $13 \%$ in the quinine $\operatorname{arm}(\mathrm{P}=0.84)$. The proportion of participants who did not adhere to quinine therapy, however, increased to $19 \%$ on the fifth day of treatment, $31 \%$ on the sixth day, and $44 \%$ on the seventh day. Only $34 \%(n=29)$ of participants in the artemether-lumefantrine group had optimal adherence whereas 51\% $(n=43)$ reported good adherence. For both groups several reasons were given for non-adherence. These included the care givers forgetting to administer the drugs (50\%, $23 / 46)$, the drugs being vomited up $(22 \%, 10 / 46)$, the child feeling better $(7 \%, 3 / 46)$, the child unable to take the drugs because of illness $(4 \%, 2 / 46)$, and the care giver not understanding dosage instructions (4\%, $2 / 46)$. Difficulty in taking the prescribed drug was reported for $32 \%(n=23)$ of participants in the quinine group compared with $19 \%(\mathrm{n}=14)$ in the artemetherlumefantrine group $(\mathrm{P}=0.03)$. Participants in the quinine group who were reported as having difficulty in taking the drugs were less likely to adhere to the study
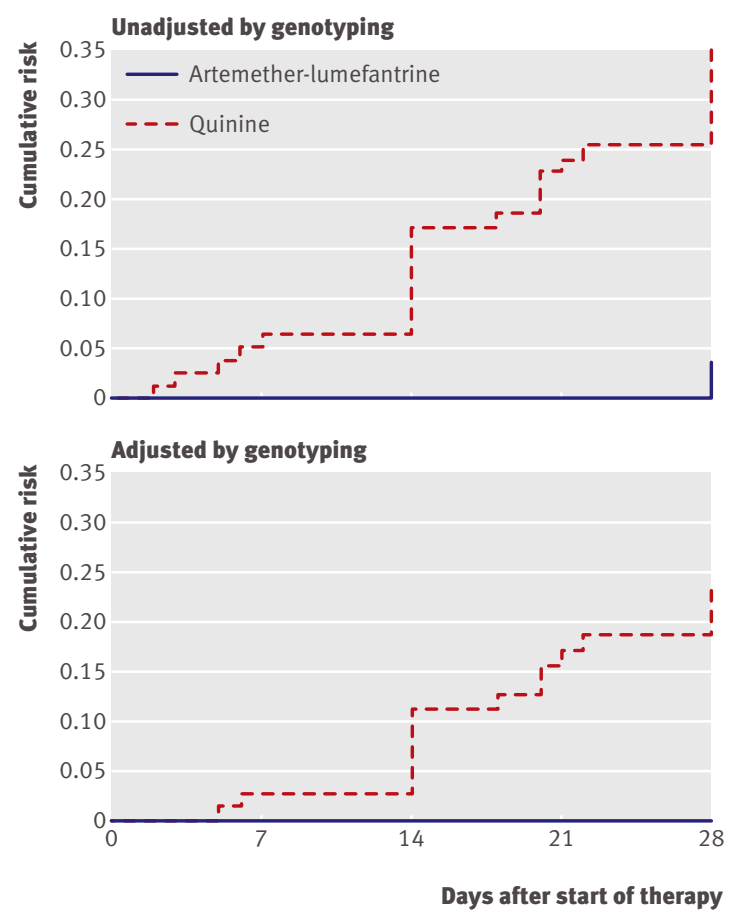

Fig 2 Kaplan-Meier curves for risk of treatment failure with oral quinine and artemether-lumefantrine in Ugandan children with uncomplicated malaria 
Table 3|Comparative effectiveness of oral quinine and artemether-lumefantrine treatment at day $\mathbf{2 8}$ in Ugandan children with uncomplicated malaria

\begin{tabular}{|c|c|c|c|c|}
\hline \multirow[b]{2}{*}{ Outcome } & \multicolumn{2}{|c|}{ Risk of failure $(95 \% \mathrm{Cl})$} & \multirow[b]{2}{*}{ Risk difference $(95 \% \mathrm{Cl})$} & \multirow[b]{2}{*}{$P$ value } \\
\hline & Quinine group $(n=86)$ & Artemether-lumefantrine group $(n=89)$ & & \\
\hline Treatment failure (\%)* & $35.3(25.6$ to 47.4$) \dagger$ & $4.1(1.3$ to 12.0$) \dagger$ & 31.3 (19.4 to 31.1) & $<0.001$ \\
\hline Treatment failure (\%) $\ddagger$ & 23.1 (14.9 to 35.0$) \S$ & $0 \S$ & 23.1 (13.2 to 33.1) & $<0.001$ \\
\hline
\end{tabular}

*Any early treatment failure, late clinical failure, or late parasitological failure.

†Unadjusted by genotyping.

$\ddagger$ Any early treatment failure, late clinical failure, or late parasitological failure caused by recrudescence.

$\S$ Adjusted by genotyping.

drugs than those that were not reported as having any difficulty; $39 \%$ (9/23) of participants who were reported as having difficulty in taking the drugs were non-adherent compared with only $21 \%$ (13/63) of those who were not reported as having any difficulty $(\mathrm{P}=0.08)$. This trend was not observed in the artemether-lumefantrine group; $7 \%(1 / 14)$ of participants who were reported as having difficulty in taking the drugs were non-adherent compared with $9 \%(7 / 75)$ who were not reported as having any difficulty $(\mathrm{P}=0.79)$. In a multivariate analysis, predictors of nonadherence included treatment with oral quinine $(\mathrm{P}<0.001)$, being male $(\mathrm{P}<0.05)$, and vomiting $(\mathrm{P}=0.02)$. Having a mean adherence of $80 \%$ or more was associated with a decreased risk of treatment failure, although this was not significant (hazard ratio 0.44, $95 \%$ confidence interval 0.19 to $1.02, \mathrm{P}=0.06$ ).

\section{DISCUSSION}

We compared the effectiveness of oral quinine with artemether-lumefantrine in the treatment of uncomplicated malaria in Ugandan children aged 6 to 59 months. To our knowledge this was the first randomised comparison of the effectiveness of these two antimalarial drugs; artemether-lumefantrine was highly effective, with only three late treatment failures (all new infections) among 74 evaluable participants (96\% efficacy) after 28 days of follow-up. In contrast, quinine was ineffective for treating uncomplicated malaria, with failure in 26 of 73 evaluable participants (64\% efficacy), including two early treatment failures and 16 late recrudescences. Participants treated with quinine were 10 times more likely to fail therapy than those treated with artemether-lumefantrine. These results have striking implications. Quinine is the most common second line therapy and is also increasingly used as first line therapy for uncomplicated malaria in Africa. Our results suggest that quinine is a poor choice for the treatment of uncomplicated malaria and that its use as first line or second line therapy for uncomplicated malaria needs to be reviewed.

Previous results for the efficacy and effectiveness of oral quinine in uncomplicated malaria have been mixed. Many studies have shown good efficacy with quinine treatment for seven days, ${ }^{11-13}$ but some have shown efficacy ${ }^{8}$ or effectiveness ${ }^{7}$ below $90 \%$, with particularly poor outcomes in studies of pregnant women. ${ }^{910}$ Several factors may have contributed to the poor effectiveness of quinine in our study. Firstly, although the quinine had been certified by the Uganda National Drug Authority as being of good quality, we did not verify this with specific tests, so that poor drug quality cannot be ruled out. Secondly, resistance to quinine may have led to treatment failures. Diminished sensitivity of cultured $P$ falciparum has been shown in Asia ${ }^{2930}$ and South America, ${ }^{31}$ but it seems to be uncommon in parasites from Africa. ${ }^{14-19}$ Thirdly, varied pharmacokinetics may have led to drug levels in some participants that were inadequate to clear parasites after seven days of treatment. However, there is little evidence for large variations in quinine pharmacokinetics. ${ }^{20}$ The fourth and probably most likely explanation for the poor effectiveness of quinine in our study was poor compliance with the dosage (three times daily for seven days). Quinine is well known for poor tolerability owing to nausea, headache, tinnitus, blurred vision, and other symptoms that increase over a few days of therapy, and poor compliance with the drug has been documented. ${ }^{21}$ In addition, even without problems of tolerability patients may stop treatment early if symptoms of malaria have resolved. Shortening the course of quinine has been proposed as a strategy to improve adherence; however, shorter courses have generally shown decreased efficacy when compared with a seven day regimen. ${ }^{632}$ In our study, multiple reasons for nonadherence were documented and poor adherence with dosing guidelines was associated with treatment failures. However, associations between adherence and outcomes were modest and not statistically significant and did not fully explain the poor effectiveness of quinine. Serum levels of quinine were not measured, and so adherence measures depended on primary care givers' reports and pill counts, which may have been inaccurate. It remains unclear if our inability to fully explain the poor effectiveness of quinine resulted from limitations in our assessment of compliance or was due to other factors.

In contrast with the results for quinine, the effectiveness of artemether-lumefantrine, the new first line drug for uncomplicated malaria in Uganda, was excellent. This result is consistent with those from other effectiveness studies, showing that unsupervised treatment with artemether-lumefantrine had the same efficacy (98\%) as supervised therapy for the treatment of uncomplicated malaria in Uganda ${ }^{33}$ and that artemether-lumefantrine had excellent effectiveness in a region of Tanzania with high rates of treatment failure with 
Table $4 \mid$ Secondary treatment outcomes at 28 days of follow-up by treatment group. Values are numbers (percentages) unless stated otherwise

\begin{tabular}{|c|c|c|c|}
\hline Outcome & $\begin{array}{l}\text { Quinine group } \\
\qquad(n=86)\end{array}$ & $\begin{array}{l}\text { Artemether-lumefantrine group } \\
\qquad(\mathrm{n}=89)\end{array}$ & $P$ value \\
\hline \multicolumn{4}{|l|}{ Adherence*: } \\
\hline None & $41(55)$ & $14(17)$ & $<0.001$ \\
\hline Difficulty taking drug & $23(31)$ & $14(17)$ & 0.03 \\
\hline \multicolumn{4}{|l|}{ Appearance of gametocytes: } \\
\hline Day 7 & $10(17)$ & $1(1)$ & 0.001 \\
\hline Day 14 & $5(8)$ & $1(1)$ & 0.07 \\
\hline Day 28 & $1(2)$ & $1(1)$ & 0.86 \\
\hline $\begin{array}{l}\text { Mean (SD) change in } \\
\text { haemoglobin concentration } \\
(\mathrm{g} / \mathrm{l}) \text {, day } 0 v \text { day } 28\end{array}$ & $12(22)$ & $13(20)$ & 0.78 \\
\hline \multicolumn{4}{|l|}{ Adverse events, days 0-28: } \\
\hline Adverse event of any severity & $21(24)$ & $16(18)$ & 0.30 \\
\hline Serious adverse event & $2(2)$ & $1(1)$ & 0.54 \\
\hline Fever & $15(71)$ & $7(44)$ & 0.49 \\
\hline Anorexia & $0(0)$ & $1(6)$ & 0.47 \\
\hline Cough & $1(5)$ & $2(13)$ & 0.74 \\
\hline Diarrhoea & $0(0)$ & $3(19)$ & 0.47 \\
\hline Recurrent seizures & $1(5)$ & $0(0)$ & 0.41 \\
\hline Repeated vomiting & $1(5)$ & $2(6)$ & 0.45 \\
\hline Rash & $1(5)$ & $0(0)$ & 0.41 \\
\hline
\end{tabular}

${ }^{\star}$ Concerns 75 children in quinine arm and 85 in artemether-lumefantrine arm.

other antimalarial drugs. ${ }^{34}$ Artemether-lumefantrine has a shorter treatment course than quinine and has excellent tolerability. However, it must be administered twice a day, ideally with a fatty meal, two factors that have led to concern about its effectiveness. Our results and those of other recent studies reassure us that, even with suboptimal adherence $(19 \%$ of participants had $<100 \%$ adherence), artemether-lumefantrine will likely perform well as a routine first line antimalarial therapy in Africa. However, other studies have shown levels of adherence to artemisinin based combination therapies lower than those in our study, ${ }^{35}$ or observed that the efficacy of unsupervised artemisinin based combination therapies was lower than that of supervised therapy, ${ }^{3637}$ emphasising the need for increased attention to education of patients and care

\section{WHAT IS ALREADY KNOWN ON THIS TOPIC}

Many malaria endemic countries recommend quinine as the second line regimen for uncomplicated malaria, to be used after failure of first line therapy

Owing to decreased efficacy of older antimalarials and limited availability of new artemisinin based combination therapies, quinine is increasingly used as the first line therapy of uncomplicated malaria in Africa

No published studies have compared the effectiveness of quinine and an artemisinin based combination therapy for uncomplicated malaria

\section{WHAT THIS STUDY ADDS}

A seven day course of quinine for the treatment of uncomplicated malaria in Ugandan children was less effective than that of artemether-lumefantrine

The recommendation for quinine as second line therapy for uncomplicated malaria in most countries in Africa is questionable givers on the appropriate dosing of new antimalarial therapies.

\section{Conclusions}

We found that the effectiveness of a seven day course of quinine for the treatment of uncomplicated malaria in Ugandan children was significantly lower than that of the new artemisinin based combination therapy artemether-lumefantrine. These results question the advisability of the recommendation for quinine as therapy for uncomplicated malaria in Africa. Rather, it seems that another artemisinin based combination therapy will be more appropriate to treat uncomplicated malaria even after initial treatment failure with an artemisinin based combination therapy. This strategy seems reasonable in Africa, as nearly all clinical failures after use of highly effective artemisinin based combination therapies (artemether-lumefantrine, artesunate-amodiaquine, and dihydroartemisinin with piperaquine) are new infections, not recrudescence. However, the use of artemisinin based combination therapies as both first line and second line therapy for uncomplicated malaria has not yet been tested in a therapeutic trial, and such evaluation should be a priority. Furthermore, the increasing use of quinine as first line therapy for uncomplicated malaria should be discouraged. It is hoped that increasing access to new drugs will allow most Africans with uncomplicated malaria to be treated promptly with artemisinin based combination therapies, the most effective available therapies for this condition.

We thank the care givers for giving consent, the children for their cooperation, the study team, and Vinay Gupta and Bryan Greenhouse for assistance with genotyping of clinical samples.

Contributors: Al devised the study, was involved in protocol development, implementation of the trial, data analysis, and drafting the manuscript. JKT devised the study, obtained funding, and was involved in protocol development and manuscript production. DK was involved in protocol development, implementation of the trial, and reviewing the manuscript. AOT devised the study, was involved in protocol development and manuscript production and is the guarantor. PJR was involved in implementation of the trial, interpretation of data, and drafting and reviewing the manuscript. GD was involved in data analysis and interpretation and reviewing the manuscript. UD’A was involved in protocol development and reviewing the manuscript. MRK and FWM were involved in reviewing the manuscript. JA and PJR wrote the first draft of the manuscript and all authors contributed to the final version of the paper.

Funding: This study received funding from the Department for International Development (DFID), UK, through the Malaria Consortium (contract No CNTR 04 5432). The sponsors of the study had no role in study design, data collection, data analysis, data interpretation or writing of this report. The corresponding author had full access to study data and had final responsibility for submission for publication. All authors reviewed the manuscript and agreed to its content.

Competing interests: None declared.

Ethical approval: This study was approved by the Makerere University Faculty of Medicine research and ethics committee and Uganda National Council for Science and Technology. All primary care givers provided written informed consent.

1 World Health Organization. Antimalarial drug combination therapy. Report of WHO technical consultation. WHO/CDS/RBM/2001.35. Geneva: WHO, 2001

2 Meshnick SR, Dobson MJ. The history of antimalarial drugs. Antimalarial chemotherapy: mechanisms of action, resistance, and new directions in drug discovery. In: Rosenthal PJ, ed. Totowa, NJ: Humana Press, 2001:15-25. 
3 World Health Organization. WHO guidelines for the treatment of malaria. WHO/HTM/MAL/2006.Geneva: WHO, 2006:1108.

4 World Health Organization.Global antimalarial drug policies database-AFRO. Antimalarial treatment policies for $\mathrm{P}$ falciparum and $P$ vivax by country in WHO Africa region. www.who.int/malaria/ amdp-afro.html (accessed 9 Sep 2008).

5 Zurovac D, Tibenderana JK, Nankabirwa J, Ssekitooleko J, Njogu NJ, Rwakimari JB, et al. Malaria case-management under artemetherlumefantrine treatment policy in Uganda. Malar / 2008;7:181.

6 Kofoed PE, Ursing J, Rodrigues A, Rombo L. Failures following initial treatment for uncomplicated malaria: quinine as second line therapy. J Pediatr Infect Dis 2007;2:121-6.

7 Adam I, Salih I, Elbashir MI. Quinine for the treatment of uncomplicated Plasmodium falciparum malaria in eastern Sudan. Trans R Soc Trop Med Hyg 2005;99:736-8.

8 Pukrittayakamee S, Chantra A, Vanijanonta S, Clemens R, Looareesuwan S, White NJ. Therapeutic responses to quinine and clindamycin in multidrug-resistant falciparum malaria. Antimicrob Agents Chemother 2000;44:2395-8.

9 McGready R, Ashley EA, Moo E, Cho T, Barends M, Hutagalung R, et al. A randomized comparison of artesunate-atovaquone-proguanil versus quinine in treatment for uncomplicated falciparum malaria during pregnancy. I Infect Dis 2005;192:846-53.

10 Adegnika AA, Breitling LP, Agnandji ST, Chai SK, Schutte D, Oyakhirome S, et al. Effectiveness of quinine monotherapy for the treatment of plasmodium falciparum malaria in pregnant women in Lambarene, Gabon. Am J Trop Med Hyg 2005;73:263-6.

11 Roche J, Guerra-Neira A, Raso J, Benito A. Surveillance of in vivo resistance of Plasmodium falciparum to antimalarial drugs from 1992 to 1999 in Malabo (Equatorial Guinea). Am J Trop Med Hyg 2003;68:598-601.

12 Ache A, Escorihuela M, Vivas E, Paez E, Miranda L, Matos A, et al. In vivo drug resistance of falciparum malaria in mining areas of Venezuela. Trop Med Int Health 2002;7:737-43.

13 Parola P, Ranque S, Badiaga S, Niang M, Blin O, Charbit JJ, et al. Controlled trial of 3-day quinine-clindamycin treatment versus 7-day quinine treatment for adult travellers with uncomplicated falciparum malaria imported from the tropics. Antimicrob Agents Chemother 2001;45:932-5.

14 Quashie NB, Duah NO, Abuaku B, Koram KA. The in-vitro susceptibilities of Ghanaian Plasmodium falciparum to antimalarial drugs. Ann Trop Med Parasitol 2007;101:391-8.

15 Menard D, Yapou F, Manirakiza A, Djalle D, Matsika-Claquin MD, Talarmin A. Polymorphisms in pfcrt, pfmdr1, dhfr genes and in vitro responses to antimalarials in plasmodium falciparum isolates from Bangui, Central African Republic. Am J Trop Med Hyg 2006;75:381-7.

16 Henry M, Diallo I, Bordes J, Ka S, Pradines B, Diatta B, et al. Urban malaria in Dakar, Senegal: chemosusceptibility and genetic diversity of plasmodium falciparum isolates. Am J Trop Med Hyg 2006;75:146-51.

17 Pradines B, Hovette P, Fusai T, Atanda HL, Baret E, Cheval P, et al. Prevalence of in vitro resistance to eleven standard or new antimalarial drugs among Plasmodium falciparum isolates from Pointe-Noire, Republic of the Congo. J Clin Microbiol 2006;44:2404-8.

18 Agnamey P, Brasseur P, Eldin de Pecoulas P, Vaillant M, Olliaro P. Plasmodium falciparum in vitro susceptibility to antimalarial drugs in Casamance (south western Senegal) during the first 5 years of routine use of artesunate-amodiaquine. Antimicrob Agents Chemother 2006;50:1531-4.

19 Tinto H, Rwagacondo C, Karema C, Mupfasoni D, Vandoren W, Rusanganwa $\mathrm{E}$, et al. In-vitro susceptibility of Plasmodium falciparum to monodesethylamodiaquine, dihydroartemisinin and quinine in an area of high chloroquine resistance in Rwanda. Trans $R$ Soc Trop Med Hyg 2006;100:509-14.

20 Pukrittayakamee S, Wanwimolruk S, Stepniewska K, Jantra A Huyakorn S, Looareesuwan S et al. Quinine pharmacokinetic pharmacodynamic relationships in uncomplicated falciparum malaria. Antimicrob Agents Chemother 2003;47:3458-63.
21 Fungladda W, Honrado ER, Thimasarn K, Kitayaporn D, Karbwang J, Kamolratanakul P, et al. Compliance with artesunate and quinine + tetracycline treatment of uncomplicated falciparum malaria in Thailand. Bull World Health Organ 1998;76(suppl 1):59-66.

22 Taylor WRJ, White NJ. Antimalarial drug toxicity: a review. Drug Saf 2004;27:25-61.

23 World Health Organization. Severe falciparum malaria. Trans $R$ Soc Trop Med Hyg 2000;94:1-90.

24 World Health Organization. WHO toxicity grading scale for determining the severity of adverse events. Geneva: WHO. www. icssc.org/Documents/Resources/

AEManual2003AppendicesFebruary_06_2003\%20final.pdf (accessed 10 Apr 2007).

25 Plowe CV, Djimde A, Bouare M, Doumbo O, Wellems TE. Pyrimethamine and proguanil resistance-conferring mutations in Plasmodium falciparum dihydrofolate reductase: polymerase chain reaction methods for surveillance in Africa. Am J Trop Med Hyg 1995;52:565-8

26 Greenhouse B, Myrick A, Dokomajilar C, Woo JM, Carlson EJ, Rosenthal PJ, et al. Validation of microsatellite markers for use in genotyping polyclonal plasmodium falciparum infections. Am J Trop Med Hyg 2006;75:836-42.

27 World Health Organization. The use of antimalarial drugs-report of a WHO informal consultation. WHO/CDS/RBM/2001.33. Geneva: WHO, 2000.

28 Fogg C, Bajunirwe F, Piola P, Biraro S, Checchi F, Kiguli J, et al. Adherence to a six-dose regimen of artemether-lumefantrine for treatment of uncomplicated Plasmodium falciparum malaria in Uganda. Am J Trop Med Hyg 2004;71:525-30.

29 Mayxay M, Barends M, Brockman A, Jaidee A, Nair S, Sudimack D, et al. In vitro antimalarial drug susceptibility and pfcrt mutation among fresh plasmodium falciparum isolates from the Lao PDR (Laos). Am J Trop Med Hyg 2007;76:245-50.

30 Chaijaroenkul W, Bangchang KN, Mungthin M, Ward SA. In vitro antimalarial drug susceptibility in Thai border areas from 1998 2003. Malar / 2005;4:37.

31 Legrand E, Volney B, Meynard B, Mercereau-Puijalon O, Esterre P. In vitro monitoring of Plasmodium falciparum drug resistance in French Guiana: a synopsis of continuous assessment from 1994 to 2005. Antimicrob Agents Chemother 2008;52:288-98.

32 Kofoed PE, Mapaba E, Lopes F, Pussick F, Aaby P, Rombo L. Comparison of 3, 5 and 7 days' treatment with Quinimax for falciparum malaria in Guinea-Bissau. Trans R Soc Trop Med Hyg 1997;91:462-4

33 Piola P, Fogg C, Bajuninwe F, Biraro S, Grandesso F, Ruzagira E, et al. Supervised versus unsupervised intake of six-dose artemetherlumefantrine for treatment of acute, uncomplicated Plasmodium falciparum malaria in Mbarara, Uganda: a randomised trial. Lancet 2005;365:1467-73.

34 Mutabingwa TK, Anthony D, Heller A, Hallett R, Ahmed J, Drakeley C, et al. Amodiaquine alone, amodiaquine+sulfadoxinepyrimethamine, amodiaquine+artesunate, and artemether-lumefantrine for outpatient treatment of malaria in Tanzanian children: a four-arm randomised effectiveness trial. Lancet 2005;365:1474-80.

35 Depoortere E, Guthmann JP, Sipilanyambe N, Nkandu E, Fermon F, Balkan S, et al Adherence to the combination of sulphadoxinepyrimethamine and artesunate in the Maheba refugee settlement, Zambia. Trop Med Int Health 2004;9:62-7.

36 Oyakhirome S, Pötschke M, Schwarz NG, Dörnemann J, Laengin M, Salazar CO, et al. Artesunate-amodiaquine combination therapy for falciparum malaria in young Gabonese children. Malar / 2007;6:29.

37 Depoortere E, Guthmann JP, Presse J, Sipilanyambe N, Nkandu E, Balkan S, et al. Efficacy and effectiveness of the combination of sulfadoxine/pyrimethamine and a 3-day course of artesunate for the treatment of uncomplicated falciparum malaria in a refugee settlement in Zambia. Trop Med Int Health 2005;10:139-45.

Accepted: 8 April 2009 